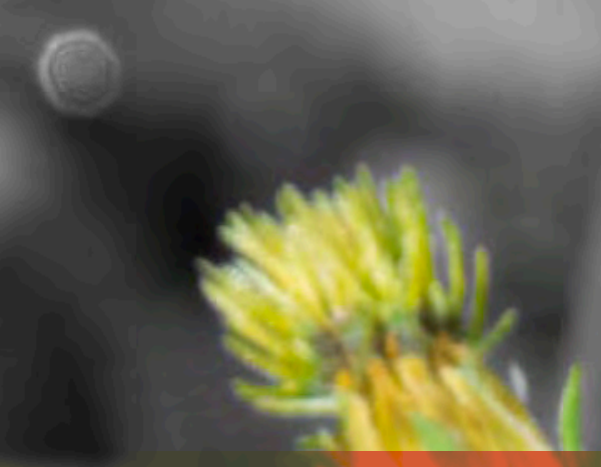

\title{
El control natural de insectos en el ecosistema cafetero colombiano
} Introducción

Pablo Benavides Machado*

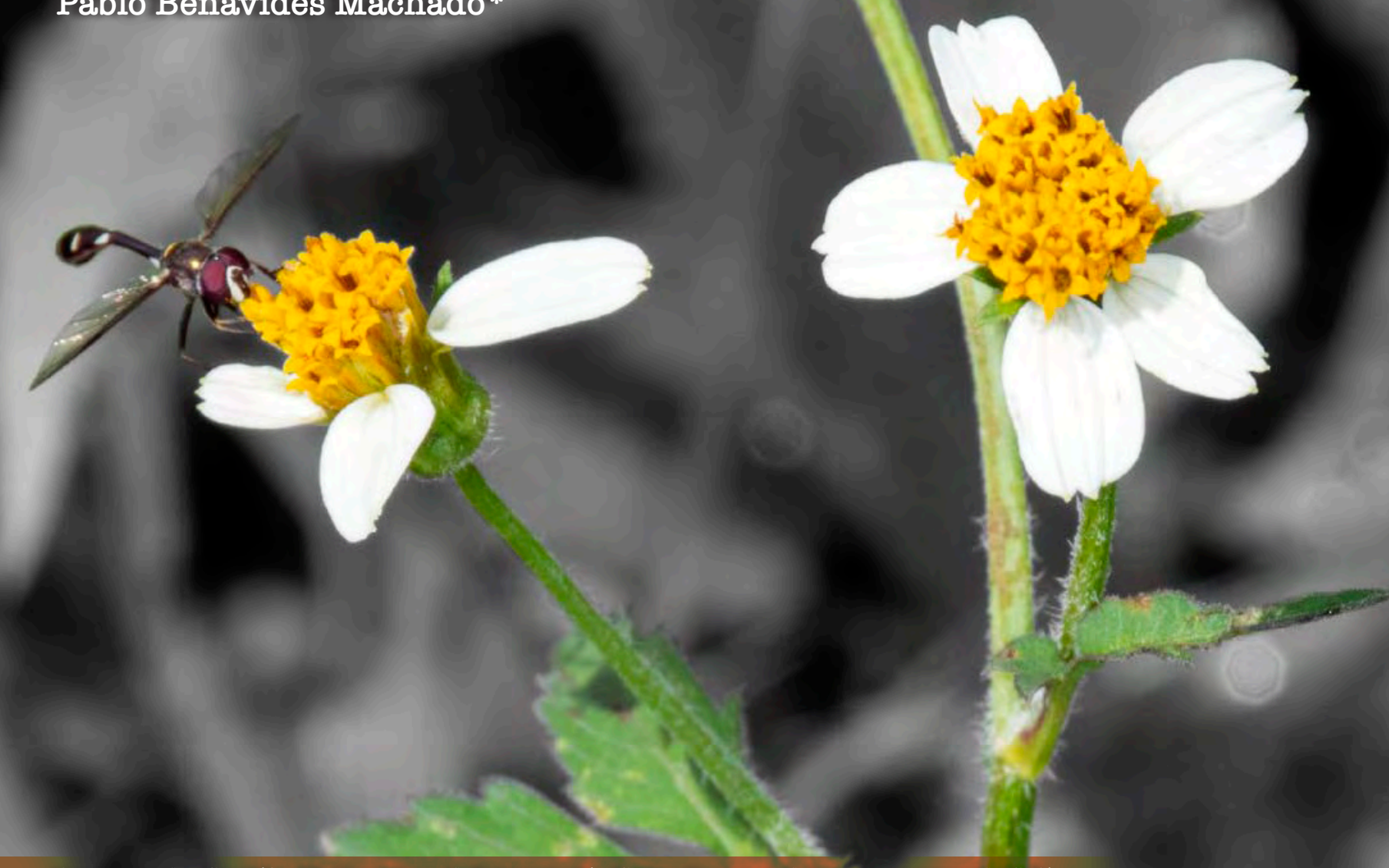

*Investigador Científico III, Disciplina de Entomología, Centro Nacional de Investigaciones de Café https://orcid.org/0000-0003-2227-4232

Como citar:

Benavides Machado, P. (2020). झl control natural en el ecosistema cafetero colombiano. En P. Benavides Machado \&e C. E. Góngora (Eds.), El Control Natural de Insectos en el Ecosistema Cafetero Colombiano (pp. 10-17). Cenicafé. https://doi.org/10.38141/10791/0001_1 
istóricamente, la Federación Nacional de Cafeteros de Colombia, a través del Centro Nacional de Investigaciones de Café - Cenicafé, ha concentrado sus esfuerzos en profundizar sobre el conocimiento de los artrópodos nativos plagas potenciales del café y la identificación de sus enemigos naturales de control, con el objetivo de evitar las intervenciones químicas para mantener los insectos plaga por debajo del nivel de daño económico, como estrategia única de manejo, de tal manera que se permita la acción del control natural por factores bióticos y abióticos. La mayoría de las plagas potenciales del café en Colombia se encuentran bajo control natural (Constantino et al., 2013), por lo tanto, se puede considerar la caficultura colombiana, no solo como uno de los cultivos agrícolas más extensos del país, sino como aquel que requiere menos pesticidas en su proceso productivo.

Paul DeBach (1964) define el control natural como la acción de factores bióticos y abióticos ambientales que mantienen una población de una plaga dentro de ciertos límites a través del tiempo, mientras que la densidad del insecto variaría con el cambio, eliminación o agregación de los componentes reguladores. Todos los organismos están bajo cierto grado de control natural, el cual fomenta al máximo la acción de los enemigos naturales que normalmente afectan una plaga (Bustillo, 2008).

Los factores bióticos y abióticos que regulan las poblaciones de los artrópodos plagas potenciales del café varían en el ecosistema cafetero colombiano; también son conocidos como factores dependientes o independientes de la densidad (Constantino, 2008) y es un modelo complejo que relaciona el sistema productivo, la altitud, la agronomía, la posición geográfica y su efecto en la biodiversidad.

La localización geográfica de la caficultura de Colombia y el manejo agronómico influyen en la diversidad de biocontroladores naturales de las plagas del café. Los reportes históricos de insectos que se alimentan de café enumeran alrededor de 136 especies para Colombia (Constantino et al., 2013); sin embargo, los 
mayores problemas de plagas que afectan la producción y calidad del café son: la broca del café Hypothenemus hampei (Ferrari) (Coleoptera: Curculionidae); el minador de las hojas del café Leucoptera coffeellum (GuérinMéneville) (Lepidoptera: Lyonetiidae); la arañita roja Oligonychus yothersi (McGregor) (Acari: Tetranychidae); la chinche de la chamusquina del café Monalonion velezangeli Carvalho \& Costa (Hemiptera: Miridae); las cochinillas de las raíces del café de las especies Puto barberi (Cockerell) (Hemiptera: Putoidae), Dysmicoccus texensis (Tinsley), Neochavesia caldasiae Balachowsky, y Pseudococcus jackbeardsleyi Gimpel \& Miller (Hemiptera: Pseudococcidae) (Benavides et al., 2013). Excepto por la broca, que fue introducida a las Américas y el Caribe desde África, los artrópodos que pueden afectar el café en Colombia son nativos, por esto, las probabilidades de encontrar agentes de control natural para estas especies son altas.

El sistema productivo y la altitud impactan las variables del clima que regulan las poblaciones de las plagas, las cuales limitan la producción y calidad del café. Con respecto al sistema productivo de café, los estudios realizados en Cenicafé (Cenicafé, 2012) demuestran que las poblaciones de la broca $H$. hampei alcanzan a duplicarse en cafetales a la sombra cuando se compara con aquellas alcanzadas en cultivos a plena exposición solar, durante los años El Niño, donde la temperatura aumenta por encima de un grado centígrado con respecto a los años Neutro (Constantino, 2010). De manera contraria, cuando se presenta un evento La Niña, las poblaciones de la plaga en los cafetales al sol son mayores que en aquellos con sombrío, siendo mínimas las cantidades de broca en este último. A pesar de lo anterior, las poblaciones del insecto en el campo son significativamente mayores cuando la temperatura aumenta. En cafetales al sol, los límites superiores e inferiores de la infestación en el campo presentan un rango menor que aquellos observados en los cafetales a la sombra. En conclusión, en cafetales a la sombra, a extremos climáticos, extremos poblacionales de la broca del café. En la práctica, controlar este insecto en sistemas agroforestales durante un evento El Niño es un reto considerable que requiere especial diligencia en la aplicación de las estrategias de control.

La altitud afecta la temperatura, existiendo una relación inversa entre ellas. Las investigaciones conducidas en Cenicafé demuestran el efecto que tiene esta variable de clima con el desarrollo de las plagas y la aparición de daños en la caficultura colombiana. Los cafetales localizados en altitudes bajas, donde la temperatura media anual supera los $21^{\circ} \mathrm{C}$, son más vulnerables al ataque de la broca, dado que se acorta el ciclo de vida del insecto, se asegura la reproducción y la fecundidad, y se aumenta la tasa de desarrollo (Giraldo et al., 2018), como consecuencia, los niveles de infestación son mayores en estas localidades (Cenicafé, 2011). Esta situación se agrava por la presencia de los eventos climáticos relacionados con El Niño, por lo que se ha calculado que, en condiciones Neutras, alrededor de 70.000 hectáreas de café son vulnerables a los efectos de la broca, mientras que con la llegada de El Niño, el área afectada supera las 400.000 hectáreas (Ramírez et al., 2014). Situaciones similares se presentan con las plagas potenciales del café tales como la arañita roja 0 . yothersi y el minador de las hojas del café L. coffeella; sin embargo, estos artrópodos tienen buen control natural influenciado por factores bióticos.

A pesar de que la broca del café es un insecto introducido a Colombia en el año 1988 (Bustillo et al., 1998), las investigaciones recientes han permitido identificar enemigos naturales nativos (Constantino et al., 2013; Gaitán et al., 2002; Vera et al., 2008). A partir de estos hallazgos se han seleccionado hongos entomopatógenos como Beauveria bassiana y Metarhizium anisopliae y se cuenta con un cepario de hongos biocontroladores de las plagas del café en Colombia (Cenicafé, 2018), depredadores tales como Cathartus quadricollis (Guérin-Méneville) y Ahasverus advena (Waltl) (Coleoptera: Silvanidae) (Laiton et al., 2018) y Solenopsis picea (Hymenoptera: Formicidae) (Cenicafé, 2017). Estos organismos son candidatos para ser usados en estrategias de control biológico por aumentación o por conservación. Los 
resultados demuestran que existe una gran biodiversidad de enemigos naturales en Colombia, que oferta controladores bióticos y contribuyen a la economía agrícola mediante procesos de adaptación ecológica.

El caso del uso de Beauveria para el control de la broca ha sido interesante, debido a que se identificaron las cepas patogénicas y más virulentas, se produjeron artesanalmente, se dieron a las casas comerciales y se usan en aspersiones inundativas, como parte de la estrategia de manejo integrado de la broca en Colombia (Góngora et al., 2009).

Con el fin de avanzar en el conocimiento sobre el minador de las hojas del café en Colombia, se realizó un estudio para diagnosticar la situación de la plaga y sus enemigos naturales en Antioquia, región de mayor producción histórica en Colombia. Para esto se realizaron cuatro muestreos aleatorios en más de 1.200 fincas (David-Rueda et al., 2016), las cuales representaron la caficultura del departamento. Se evidenció en esta investigación que el minador está bajo control natural, con porcentajes de la plaga inferiores a 3\% y parasitismos naturales que oscilaron entre $58 \%$ y $89 \%$. Se identificaron siete especies de parasitoides de la familia Eulophidae, siendo Closterocerus coffeellum Ihering (Hymenoptera: Eulophidae) la responsable de más del $80 \%$ del parasitismo natural. Estos resultados soportan la necesidad de evitar los insecticidas químicos para el control de esta plaga en Colombia, dado que se agravarían los problemas y comenzaría una dependencia innecesaria al uso de pesticidas químicos. Se recomienda así, realizar limpieza de los platos de los árboles de café jóvenes, de menos de dos años, al momento del inicio de los períodos secos, el establecimiento de un manejo integrado de arvenses en las calles del cafetal y la eliminación de los insecticidas de síntesis química (Benavides et al., 2013). Con esta recomendación se asegura la presencia suficiente de flores en el cafetal que atraen y alimentan los eulófidos controladores naturales, se garantiza la sobrevivencia de los mismos y se mantienen los niveles de parasitismo de las larvas de minador (Figura 1).
La chinche de la chamusquina del café, una plaga inducida en café que ataca principalmente cultivos de cacao, guayaba y aguacate, posee un número importante de enemigos naturales, entre los cuales se describen varias especies de chinches asesinas de la familia Reduviidae (Giraldo et al., 2011), un género de entomopatógeno de reciente descripción, Clonostachyssp. (Ramírez etal., 2008) y una cepa específica de B. bassiana (Cenicafé, 2017). Los resultados actuales del éxito de estos organismos en el control de Monalonion en el campo sugieren su uso en estrategias de control biológico por aumentación y por conservación. Un valor agregado de la generación de este conocimiento radica en el hecho de que los caficultores del departamento de Huila presionan para que se use el control biológico y natural. En el futuro cercano se estarían suministrando organismos para la recuperación de los enemigos naturales nativos como una forma de recuperación ecológica de ambientes modificados.

Tanto la arañita roja como la gran mayoría de las plagas del café son presa de varios depredadores identificados en los cafetales colombianos (Gil et al., 2014; Giraldo et al., 2011). El reto que persiste con el uso de los depredadores es que, por ser generalistas y no depender exclusivamente de los artrópodos plagas para asegurar su sobrevivencia, podrían desplazar otras especies de insectos no blanco o causar desequilibrios ecológicos en los sistemas de producción cafetero. Su introducción en estrategias de aumentación está en discusión, pero su utilidad en la restauración de ambientes modificados es una mejor alternativa al uso de pesticidas químicos.

El hallazgo de parasitoides de las cochinillas de las raíces del café (Cenicafé, 2014), y la relación que existe entre la plaga con hormigas y hongos simbiontes (Villegas \& Benavides, 2011) evidencian la existencia de interacciones entre: 1. la planta de café, 2. el suelo donde crecen las raíces, 3 . la cochinilla de las raíces, 4. las hormigas que transportan y acompañan a las cochinillas, 5. los hongos endosimbiontes que protegen a las especies de los géneros Dysmicoccus y Pseudococcus, 6. Ias avispas parasitoides de la familia Encyrtidae, y 7 . el 


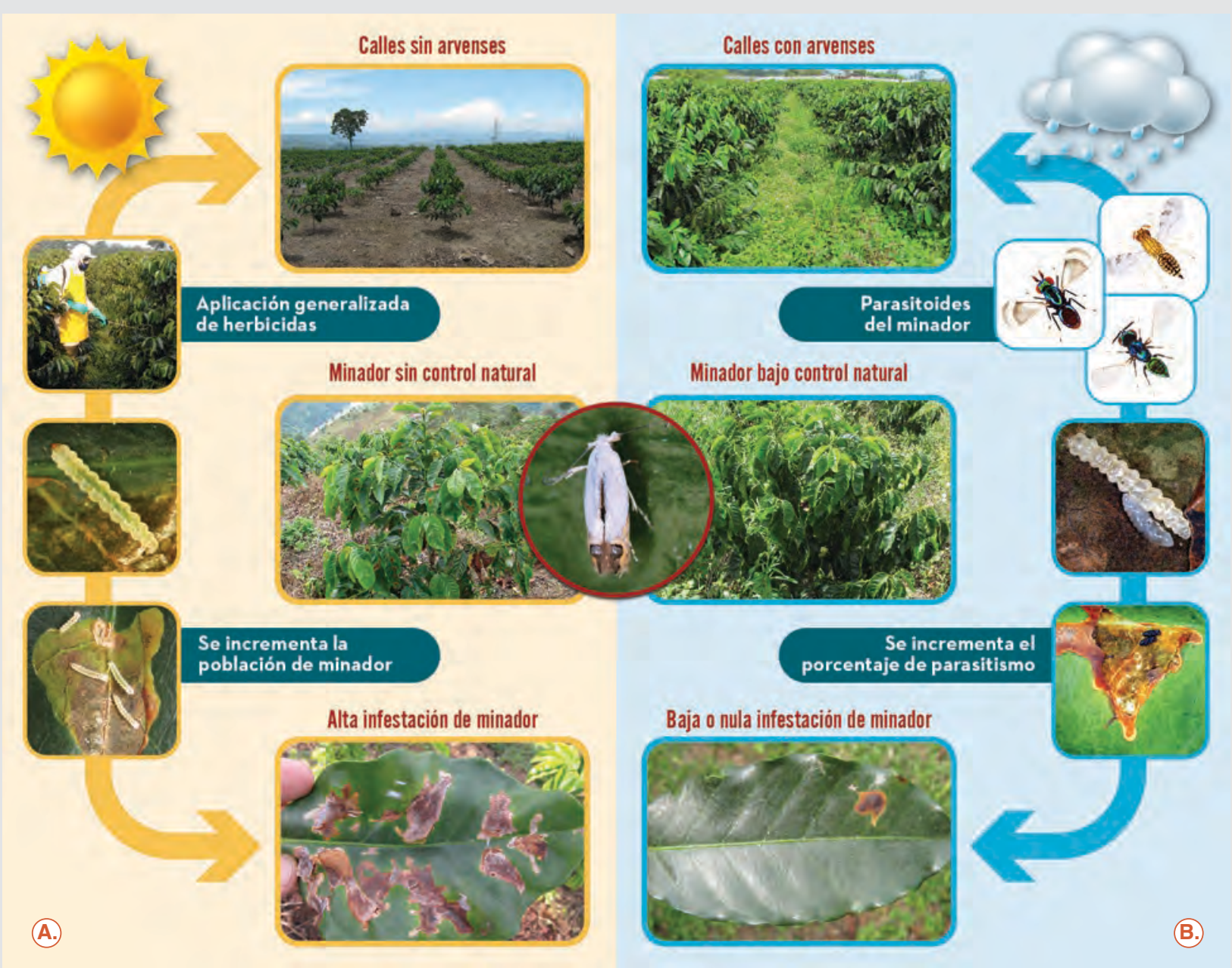

Figura 1.

Esquema de factores que A. favorece la presencia y ataque del minador de las hojas del café y B. benefician la presencia y acción de los enemigos del minador y permiten el control natural.

clima (Figura 2). Dadas las características de la plaga de atacar raíces y encontrarse en el suelo, se tiene la hipótesis de que los enemigos naturales deben perseguir señales químicas provenientes de alguno de los organismos que conforman este sistema. Esta relación muestra un esquema que explica la complejidad de las relaciones ecológicas que acompañan los ecosistemas cafeteros. El estudio de cada organismo, de manera individual, difícilmente conllevaría a hallar las soluciones que requiere la caficultura para enfrentar esta plaga.

En la última década, la Disciplina de Entomología de Cenicafé ha participado en la descripción de tres insectos plagas, nuevas especies para la ciencia, se aumentó la lista de especies asociadas a la rizosfera del café a 23 y se emitieron recomendaciones para 25 especies de insectos. De no ser por el control natural, estas especies de insectos pudieron haberse convertido en plagas de importancia económica. En la mayoría de los casos donde estos insectos realizaron daños en café se habían aplicado insecticidas piretroides de manera generalizada, eliminando la fauna benéfica de las plagas secundarias; se había eliminado la cobertura del suelo, alejando de la plantación a los enemigos naturales de las plagas potenciales; o se habían eliminado bosques o matorrales cercanos, que 
obligaron a los insectos a emigrar hacia áreas y cultivos aledaños. El manejo de estos problemas esporádicos requiere de recomendaciones que protejan la fauna benéfica y restauren el equilibrio en el ecosistema. No se recomienda usar insecticidas de amplio espectro para atender ninguno de estos problemas inducidos y transitorios.

Los registros recientes de enemigos naturales que controlan insectos plagas potenciales en café y cultivos asociados son la muestra de que la biodiversidad en los ecosistemas cafeteros provee un servicio ecosistémico importante. La identificación de especies de parasitoides de Anastrepha fraterculus, insecto abundante en las cerezas de café que no causan daños económicos al cultivo (Cenicafé, 2011; Giraldo et al., 2015), son un ejemplo de que la caficultura provee de enemigos naturales a esta especie, plaga limitante de los cultivos de frutas de la región cafetera colombiana. El valor económico de este aporte es difícil de medir. Los listados recientes de enemigos naturales en la caficultura colombiana superan las 40 especies (Constantino et al., 2013). El reto actual es aumentar el conocimiento sobre estos insectos enemigos naturales para poder valorarlos y usarlos en estrategias novedosas de control, eficaces y más limpias.

Son ejemplos de control natural, la acción que ejercen las hormigas Crematogaster spp., Leptothorax spp., Pheidole spp., Pseudomyrmex spp. y Solenopsis, como depredadores de larvas del pasador de las ramas del café, Xylosandrus morigerus. El control del gusano trozador y descortezador del tallo de café Spodoptera frugiperdapor las avispas parasitoides de huevos Telenomus remus y Trichogramma atopovirilia, y los parasitoides de larvas Chelonus insularis, Archytas sp., Voria sp. y Winthemia sp. Las avispas del género Conura sp. parasitando las larvas de gusano gelatina Paracraga argentea. El parasitismo de la polilla de los racimos de café Pococera hermasalis por parasitoides de la familia Braconidae y depredadores Pentatomidae. El control natural de la cochinilla harinosa de las ramas del cafeto Planococcus citri por los coccinélidos Cryptolaemus montrouzieri, Harmonia axyridis, Tenuisvalae

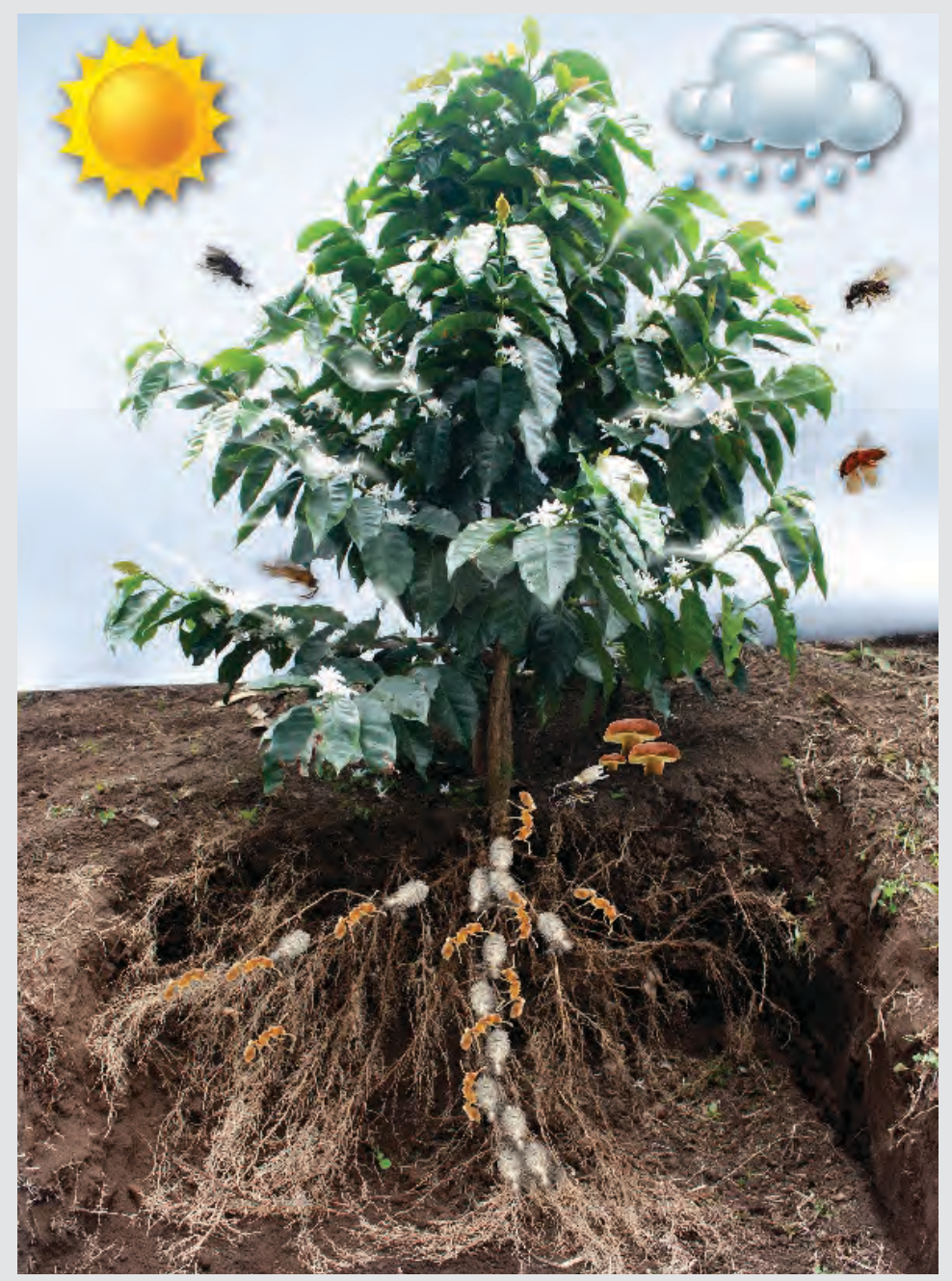

Figura 2.

Interacción de factores involucrados en los ataques de las cochinillas de las raíces del café y su posible relación con la ecología química. notata y Diomus sp.; así como parasitoides de la familia Encyrtidae (Constantino et al., 2013).

Por lo anterior, se requiere mantener esfuerzos de investigación en la biología básica de los insectos herbívoros del café y la búsqueda de los enemigos naturales que los controlan. Las alternativas químicas deben continuar siendo las últimas a considerar en un programa de manejo integrado, recayendo la responsabilidad actual en propender por la producción estable y creciente de bienes agrícolas, procurando la sostenibilidad del medio ambiente, evitando los efectos nocivos en la flora y fauna, asegurando el bienestar de los agricultores y cuidando de la salud humana.

El Control Natural de Insectos en el Ecosistema Cafetero Colombiano 


\section{Literatura citada}

Benavides Machado, P., Gil Palacio, Z. N., Constantino, L. M., Villegas García, C., \& Giraldo Jaramillo, M. (2013). Plagas del café. Broca, minador, cochinillas harinosas y monalonion. En Federación Nacional de Cafeteros de Colombia (Ed.), Manual del cafetero colombiano: investigación y tecnología para la sostenibilidad de la caficultura (Vol. 2, pp. 215-260). Cenicafé.

Bustillo-Parley, A. E. (2008). Cómo implementar un programa de manejo integrado de plagas. En A. E. Bustillo Parley (Ed.), Los insectos y su manejo en la caficultura colombiana (pp. 94-109). Cenicafé.

Bustillo-Parley, A. E., Cardenas, R., Villalba, D. A., Benavides Machado, P., Orozco, J., \& Posada, F. J. (1998). Manejo integrado de la broca del café: Hypothenemus hampei (Ferrari) en Colombia. Cenicafé. http://hdl.handle.net/10778/848

Centro Nacional de Investigaciones de Cáfe. (2011). Informe anual Cenicafé 2011. https://www.cenicafe. org/es/publications/Informe anual completo.pdf

Centro Nacional de Investigaciones de Cáfe. (2012). Informe anual Cenicafé 2012. https://www.cenicafe. org/es/publications/Informe 2012.pdf

Centro Nacional de Investigaciones de Cáfe. (2014). Informe anual Cenicafé 2014. https://www.cenicafe. org/es/publications/informeAnualCenicafe2014.pdf

Centro Nacional de Investigaciones de Cáfe. (2017). Informe anual Cenicafé 2017. https://www.cenicafe. org/es/publications/Informe2017.pdf

Centro Nacional de Investigaciones de Cáfe. (2018). Informe anual Cenicafé 2018. https://www. cenicafe. org/es/publications/Informe2018.pdf

Constantino, L. M. (2008). El control natural de los insectos. En A. E. Bustillo Parley (Ed.), Los insectos y su manejo en la caficultura colombiana (pp. 110-113). Cenicafé.

Constantino, L. M. (2010). La broca del café... un insecto que se desarrolla de acuerdo con la temperatura y la altitud. Brocarta, 39, 1-2. https://www.cenicafe.org/es/publications/brc039.pdf

Constantino, L. M., Gil-Palacio, Z., Benavides Machado, P., Martínez, H., Giraldo-Jaramillo, M., \& Villegas G., C. (2013). Otros habitantes naturales del cafetal. En Federación Nacional de Cafeteros de Colombia (Ed.), Manual del cafetero colombiano: investigación y tecnología para la sostenibilidad de la caficultura (Vol. 2, pp. 261-306). Cenicafé.

David-Rueda, G., Constantino, L. M., Montoya, E. C., Ortega, O. E., Gil Palacio, Z. N., \& Benavides Machado, P. (2016). Diagnóstico de Leucoptera coffeella (Lepidoptera: Lyonetiidae) y sus parasitoides en el departamento de Antioquia, Colombia. Revista Colombiana de Entomología, 42(1), 4-11. https://doi. org/10.25100/socolen.v42i1.6662

DeBach, P. (1964). Biological Control of Insect Pests and Weeds. Reinhold Publishing Corporation. 
Gaitán, A., Valderrama, A. M., Saldarriaga, G., Vélez, P., \& Bustillo-Parley, A. E. (2002). Genetic variability of Beauveria bassiana associated with the Coffee Berry Borer Hypothenemus hampei and other insects. Mycological Research, 106(11), 1307-1314. https://doi.org/10.1017/S0953756202006676

Gil-Palacio, Z., Constantino, L. M., Martínez, H., \& Benavides Machado, P. (2013). Aprenda a manejar la arañita roja del café. Avances Técnicos Cenicafé, 436, 1-8. http://hdl.handle.net/10778/473

Giraldo-Jaramillo, M., Benavides Machado, P., Rendón, J. R., Montoya, D. F., Farfán-Valencia, F., \& Trejos Pinzón, J. F. (2015). Las moscas de las frutas de la zona central cafetera de Colombia y su clave ilustrada. Revista Cenicafé, 66(2), 58-72. http://hdl.handle.net/10778/655

Giraldo-Jaramillo, M., Galindo-Leva, L. A., Benavides Machado, P., \& Forero, D. (2011). Aprenda a conocer las chinches depredadoras de plagas del café. Avances Técnicos Cenicafé, 412, 1-8. http://hdl. handle.net/10778/338

Giraldo Jaramillo, M., García, A., \& Parra, J. R. (2018). Biology, thermal requirements, and estimation of the number of generations of Hypothenemus hampei (Ferrari, 1867) (Coleoptera: Curculionidae) in the State of São Paulo, Brazil. Journal of Economic Entomology, 111(5), 2192-2200. https://doi.org/10.1093/jee/ toy 162

Góngora, C. E., Marín-Marín, P., \& Benavides Machado, P. (2009). Claves para el éxito del hongo Beauveria bassiana como controlador biológico de la broca del café. Avances Técnicos Cenicafé, 384, 1-8. http://hdl.handle.net/10778/346

Laiton, L. A., Constantino, L. M., \& Benavides Machado, P. (2018). Capacidad depredadora de Cathartus quadricollis y Ahasverus advena (Coleoptera: Silvanidae) sobre Hypothenemus hampei (Coleoptera: Curculionidae) en laboratorio. Revista Colombiana de Entomología, 44(2), 200-205. https://doi.org/10.25100/ socolen.v44i2.7319

\section{Como citar:}

Benavides Machado, P. (2020). El control natural en el ecosistema cafetero colombiano. En P. Benavides Machado \& C. E. Góngora (Eds.), El Control Natural de Insectos en el Ecosistema Cafetero Colombiano (pp. 10-17). Cenicafé. https://doi.org/10.38141/10791/0001 1 